\title{
Regulatory elements in the FBP1 promoter respond differently to glucose- dependent signals in Saccharomyces cerevisiae
}

\author{
Oscar ZARAGOZA, Olivier VINCENT ${ }^{1}$ and Juana M. GANCEDO ${ }^{2}$ \\ Instituto de Investigaciones Biomédicas "Alberto Sols", CSIC-UAM, Arturo Duperier 4, E-28029 Madrid, Spain
}

In Saccharomyces cerevisiae expression of the fructose-1,6bisphosphatase-encoding gene, $F B P 1$, is controlled by glucose through the upstream activating sequences UAS1 and UAS2 and the upstream repressing sequence URS1 in its promoter. We have studied the regulation of the proteins that could bind to these elements. We have investigated the role of the putative transcription factors Cat8 and Sip4 in the formation of specific DNA-protein complexes with UAS1 and UAS2, and in the expression of UAS1-lac $Z$ and UAS2-lacZ. The expression of CAT8-lacZ and SIP4-lacZ has been also measured in mig1, tup1 or $h x k 2$ mutants, partially refractory to catabolite repression. We conclude that there is no strict correlation between Cat 8 and Sip4 expression or in vitro formation of DNA-protein complexes and expression of UAS1-lacZ and UAS2-lacZ. The URS1 element binds the regulatory protein Mig1, which blocks tran- scription by recruiting the proteins Cyc 8 and Tup1. The pattern of complexes of URS1 with nuclear extracts was dependent on the carbon source and on Cyc8, but not on Tup1; it was also affected by the protein kinase Snf1 and by the exportin Msn5. The repression caused by URS1 in a fusion gene was dependent on Mig1, Cyc8 and Tup1, and on the carbon source in the medium; in a snfl strain the repression observed was independent of the carbon source. Expression of Mig1 could occur in the absence of Snf1 and was moderately sensitive to glucose. We present data showing that different elements of the regulatory system controlling FBPI responded differently to the concentration of glucose in the medium.

Key words: catabolite repression, fructose-1,6-bisphosphatase, signalling, transcription factor, yeast.

\section{INTRODUCTION}

In Saccharomyces cerevisiae the rate of transcription of a great variety of genes is modified in response to changes in the carbon source available to the yeast [1-3]. The alterations in transcription are mediated by the interplay of activators and repressors that interact with distinct regions in the promoters of the regulated genes [4].

A number of genes, encoding enzymes required for the growth of yeast on gluconeogenic carbon sources, share a common upstream activating sequence (UAS) in their promoters. This UAS has been called carbon-source-responsive element (CSRE), due to its ability to direct carbon-source-regulated expression of fusion genes [5-7]. The FBPI gene, which encodes fructose-1,6bisphosphatase, is unique among the gluconeogenic genes in possessing, in addition to a CSRE (UAS2), a second element, UAS1, which includes a sequence similar to CSRE but which does not bind the same nuclear proteins $[6,8]$. The protein(s) binding UAS1 have not been yet identified, but they appear to be repressed by glucose $[6,8]$. In the case of the CSRE, two zinccluster proteins, Cat8 and Sip4, are able to bind the DNA $[9,10]$.

In addition to the two UAS elements, the FBPI promoter contains an upstream repressing sequence (URS) able to bind the regulatory protein Mig1 [11,12]. Relief from inhibition by Mig1, which takes place in the absence of glucose, is dependent on the protein kinase Snf1 $[13,14]$, which is also required for the operation of both UAS1 and UAS2 [6,7].

The changes in the operativity of the regulatory factors that bind to the different control elements in the FBP1 promoter should depend on a signal-transduction pathway which responds to the presence of glucose. Although it has been shown that
cAMP is able to repress the expression of the FBP1 gene, this signal is redundant with other control mechanisms [15]. The glucose sensors $\mathrm{Snf} 3$ and Rgt2 are also dispensable for catabolite repression of $F B P 1$ [16].

To investigate further the mode of regulation of UAS1, UAS2 and URS1, we have analysed the capacity of the corresponding DNA sequences to form DNA-protein complexes with nuclear proteins from different mutants, and measured in parallel the levels of expression of UAS1-lacZ, UAS2-lacZ and CYClURS1-lacZ (where $C Y C 1$ encodes cytochrome $c$ ) in the same mutants and under different metabolic conditions. We have also examined different factors which may affect the expression of Cat8, Sip4 and Mig1.

We conclude that there is no strict correlation between the expression of the genes encoding proteins which bind the regulatory elements UAS1, UAS2 or URS1 and that of reporter genes under the control of these elements. This is likely to be due to the fact that both the amount and the activity of the corresponding proteins are subject to control by glucose.

\section{EXPERIMENTAL}

\section{Yeast strains and growth conditions}

Yeast strains used in this study are listed in Table 1. The yeasts were grown at $30{ }^{\circ} \mathrm{C}$ in YPD $(1 \%$ yeast extract $/ 2 \%$ peptone $/ 2 \%$ glucose) or in Difco yeast nitrogen base (YNB) with the carbon source(s) indicated in each case and collected at the exponential phase of growth $(2-3 \mathrm{mg}$ of wet weight $/ \mathrm{ml})$. When the yeasts were grown in the presence of $0.2 \%$ glucose they were collected when there was still glucose present in the medium (4-7 mM). Unless indicated otherwise, to obtain derepressed cells yeasts

Abbreviations used: CSRE, carbon-source-responsive element; URS, upstream repressing sequence; UAS, upstream activating sequence; YNB, yeast nitrogen base.

1 Present address: Centro de Investigaciones Biológicas, CSIC, Velázquez 144, 28006 Madrid, Spain.

2 To whom correspondence should be addressed (e-mail jmgancedo@iib.uam.es). 
Table 1 Yeast strains used in this study

\begin{tabular}{lll}
\hline Strain & Relevant genotype & Source/Reference \\
\hline W303-1A & MATa ade2-1 his3-11,15 leu2-3,112 trp1-1 ura3-1 & {$[48]$} \\
S288C & MATa SUC2 gal2 mal CUP1 & {$[49]$} \\
MCY4026 & MATa ade2-1 his3-11,15 leu2-3,112 trp1-1 ura3-1 sip4::LEU2 & {$[10]$} \\
MCY4029 & MATa ade2-1 his3-11,15 leu2-3,112 trp1-1 ura3-1 cat8:: TRP1 & {$[10]$} \\
HB190 & MATa ade2-1 his3-11,15 leu2-3,112 trp1-1 ura3-1 mig1::LEU2 & {$[36]$} \\
WHC-3A & MATa ade2-1 his3-11,15 leu2-3,112 trp1-1 ura3-1 1 hxk2::LEU2 & {$[32]$} \\
H366 & MATa ade2-1 his3-11,15 leu2-3,112 trp1-1 ura3-1 snf1::HIS3 & {$[36]$} \\
CJM207 & MATa ade2-1 his3-11,15 leu2-3,112 trp1-1 ura3-1 cyc8::LEU2 & This study \\
L0Z021 & MATa ade2-1 his3-11,15 leu2-3,112 trp1-1 ura3-1 tup 1::HIS3 & This study \\
PAY20 & MATa ade2-1 his3-11,15 leu2-3,112 trp1-1 ura3-1 msn5::HIS3 & {$[50]$}
\end{tabular}

grown on glucose were washed twice with distilled water, suspended at $20 \mathrm{mg} / \mathrm{ml}$ in YP $(1 \%$ yeast extract $/ 2 \%$ peptone $)$ containing $2 \%$ ethanol and incubated overnight at $30{ }^{\circ} \mathrm{C}$. In some cases the yeasts were grown in a synthetic complete medium [17] with the carbon sources indicated in the corresponding Figure.

\section{Plasmids}

Plasmid pJJ11b is a centromeric plasmid with the lac $Z$ gene under the control of the FBP1 promoter [6]. Plasmids containing UAS1-lac $Z$ and UAS2-lac $Z$ fusion genes have been described previously [8], as well as pDG225 containing a $C A T 8$-lac $Z$ fusion gene [7] and pSIP4lacZ [18]. Plasmid pLG669-ZS, containing a CYC1-lacZ fusion gene, was derived from pLG669-Z [19] by removal of a $S m a \mathrm{I}-S m a \mathrm{I}$ fragment of $1.4 \mathrm{~kb}$. Plasmid pOV31 contains the URS1 sequence $(-184$ to -201$)$ from the FBP1 promoter inserted in the SmaI site of pLG669-ZS.

pDSB [20] was used to disrupt the $C Y C 8$ gene in a W303-1A strain, yielding strain CJM207. pTUP1::HIS3 was constructed by cloning a $1 \mathrm{~kb}$ SmaI-SalI fragment from YDp-H [21] into the StuI and SalI sites from plasmid pFW47 [22]. This plasmid was digested with $P v u I I$ and the corresponding $2.2 \mathrm{~kb}$ fragment was used to disrupt the TUP1 gene in a W303-1A strain, yielding strain LOZ021.

Cloning procedures were performed according to standard protocols. Yeast cells were transformed using the lithium acetate method.

The different mutants used, mig1, tup 1, hxk2, snf1, cat 8 and sip4, are deletion mutants with part of the open reading frame deleted. The exact constructions are described in the references given in Table 1.

\section{Northern-blot analysis}

Total RNA was extracted as described in [23] using the Gibco TRIzol reagent. The RNA samples were heated at $65^{\circ} \mathrm{C}$ for $15 \mathrm{~min}$, fractionated on $1.5 \%$ agarose gels containing $2.2 \mathrm{M}$ formaldehyde and transferred to a nylon membrane. The membrane was stained with $0.02 \%$ Methylene Blue in $0.3 \mathrm{M}$ sodium acetate, $\mathrm{pH} 4.3$, and then washed with water to visualize the rRNAs. The membrane was destained with $1 \%$ SDS and hybridization was performed in a buffer containing $50 \mathrm{mM}$ $\mathrm{Na}_{2} \mathrm{HPO}_{4}(\mathrm{pH} 7.2), 1 \mathrm{mM}$ EDTA, $1 \%$ BSA and $7 \%$ SDS. As probe we used a $0.56 \mathrm{~kb}$ StyI-StyI fragment of the $M I G 1$ gene, from position +820 to +1381 . The probe was labelled as in [24] using the Amersham Pharmacia Biotech labelling kit. To quantify the RNA the gels were autoradiographed, scanned, and the area and intensity of the bands measured with the appropriate software (NIH image program).

\section{Preparation of extracts and enzymic tests}

Yeast extracts were prepared in $20 \mathrm{mM}$ imidazole buffer, $\mathrm{pH} 7$, by shaking with glass beads; for $\beta$-galactosidase assays the centrifugation step was omitted. $\beta$-Galactosidase was assayed as in [25], with the samples being centrifuged before reading. Fructose1,6-bisphosphatase was tested spectrophotometrically as described in [26]. Protein was determined in the crude extracts using the bicinchoninic acid (BCA) protein assay (Pierce), with BSA as a standard.

\section{Preparation of yeast nuclear extracts and band-shift assays}

Nuclear extracts were obtained as described in [27]. Band-shift assays were performed as described previously, using oligonucleotides OL1 and OL2, which correspond to UAS2 ${ }_{F B P 1}$ and UAS1 $1_{F B P 1}$ respectively [6], or oligonucleotide URS1, which includes a fragment $(-201$ to -184$)$ from the $F B P 1$ promoter:

$$
\begin{aligned}
& \text { 5'-tcgaCtTCCCCACACTATATTAg-3' } \\
& \text { 3'-GAAGGGGTGTGATATAATcagct-5' }
\end{aligned}
$$

\section{RESULTS}

\section{Cat8 and Sip4 play different roles in the regulation of UAS1 and UAS2}

It has been reported previously that the transcription factor Cat8 is required for the formation of DNA-protein complexes with CSRE elements $[28,29]$ and for transcription directed by such elements [30]. Sip4, a $\mathrm{C}_{6}$ zinc-cluster protein related to Cat8, may also contribute to transcriptional activation by CSRE [10]. To examine whether Cat8 and/or Sip4 are required for the formation of DNA-protein complexes between UAS1 or UAS2 from FBPI and nuclear proteins, we performed band-shift experiments. The nuclear extracts were prepared either from wild-type cells or from cat8 or sip4 mutants grown on glucose or derepressed overnight in $\mathrm{YP} /$ ethanol. As shown in Figure 1, no specific complexes were observed when extracts from repressed cells were used. With derepressed cells, complexes with UAS1 were still formed with extracts from cat 8 or sip 4 mutants, although in the case of the sip 4 mutant the amount of the low-mobility complex was strongly reduced. In contrast, the complexes formed with the UAS2 element were completely dependent on both Cat 8 and Sip4.

To study whether the formation of complexes in vitro reflects the operativity of the UASs in vivo, we tested the expression of the fusion genes FBP1-lacZ, UAS1-lacZ and UAS2-lac Z in cat 8 and sip 4 mutants. As shown in Figure 2, under derepressed conditions, the lack of Sip4 had no significant effect on the 
A
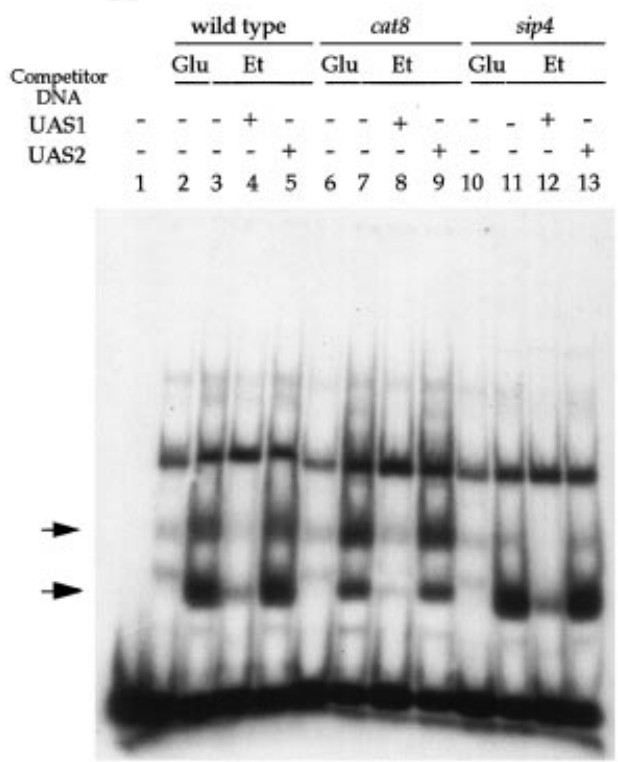

B

\begin{tabular}{|c|c|c|c|c|c|c|c|c|}
\hline \multicolumn{4}{|c|}{ wild type } & \multicolumn{3}{|c|}{ cat8 } & \multicolumn{2}{|r|}{$\operatorname{sip} 4$} \\
\hline Glu & & & & Glu & Et & & Glu & Et \\
\hline - & & & + & - & - & + & - & - \\
\hline- & & & - & - & - & & - & -+ \\
\hline 2 & 3 & & 5 & 6 & 7 & & 10 & \\
\hline
\end{tabular}

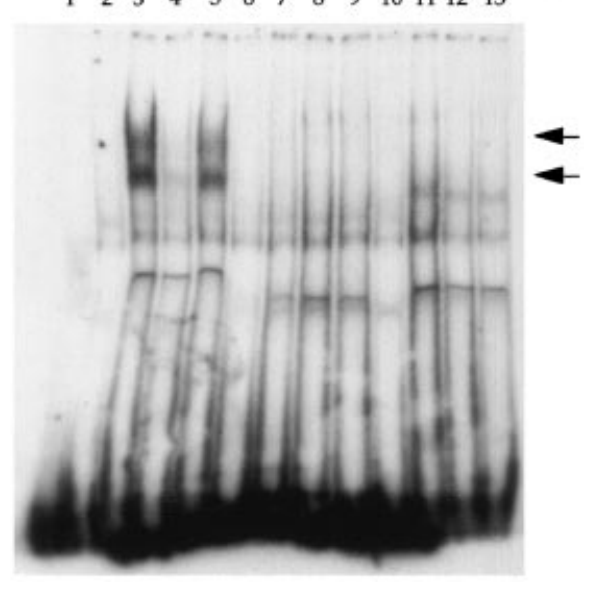

Figure 1 Effect of the cat8 and sip4 mutations on the DNA-protein complexes formed with UAS sequences from FBP1

Nuclear protein extracts were prepared from wild-type, cat8 or sip4 strains grown on YPD (Glu) or derepressed in YP/ethanol (Et). (A) The oligonucleotide corresponding to UAS1 ${ }_{F B P 1}$ was used as probe, and $20 \mu \mathrm{g}$ of nuclear proteins was added to the samples in lanes $2-13$. (B) The oligonucleotide corresponding to UAS2 ${ }_{F B P 1}$ was used as probe and $40 \mu \mathrm{g}$ of nuclear protein was added to the samples in lanes 2-13. Where indicated, a 100-fold excess of competitor DNA was used. See the Experimental section for further details. Specific complexes are indicated by arrows.

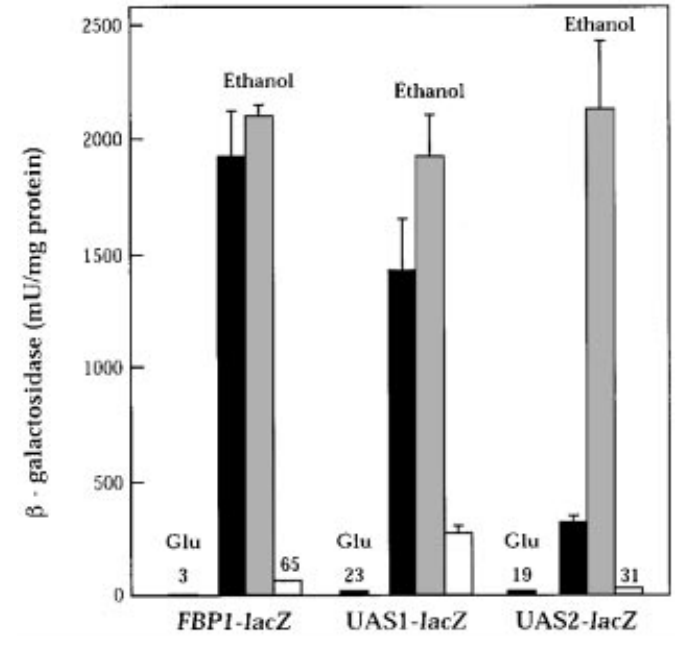

Figure 2 Effect of deletions in the SIP4 or CAT8 genes on the expression of fusion genes

Yeasts with different mutations (wild type, black bars; sip4, grey bars; cat8, white bars), transformed with plasmids containing the fusion genes indicated, were grown in YNB/glucose or derepressed in YP/ethanol as described in the Experimental section (which also contains a description of the plasmids and recipient strains used). Data are means \pm S.D. from at least three independent experiments.

transcription driven by the $F B P 1$ promoter or by UAS1 and, surprisingly, had a strong activating effect on the transcription directed by UAS2. On the other hand, in cat 8 mutants, the expression of FBP1-lac $Z$ and UAS2-lac $Z$ was strongly repressed. Expression of UAS1-lac $Z$ was significant, but reached

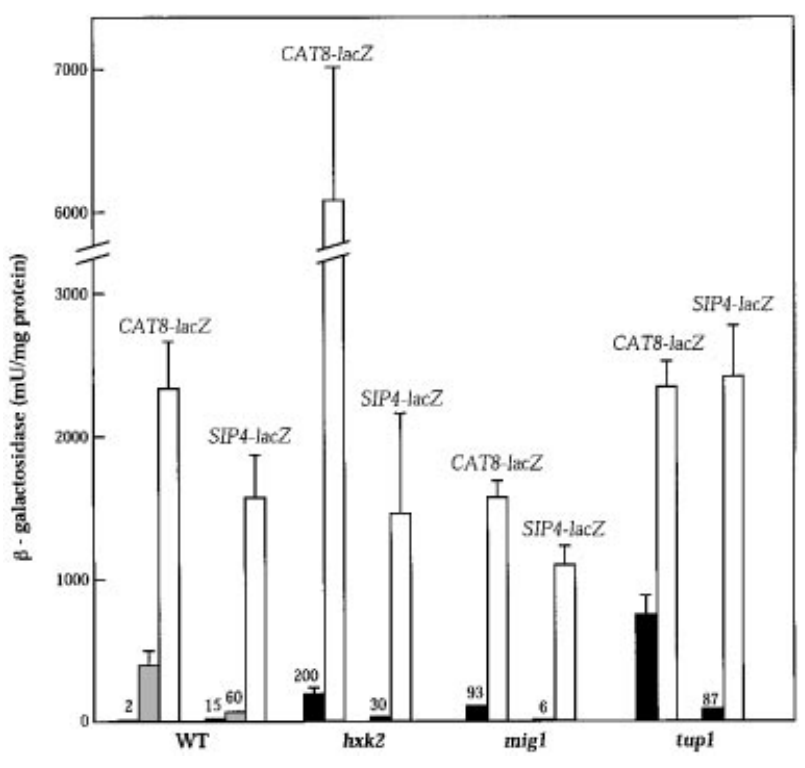

Figure 3 Expression of the fusion genes CAT8-lacZ and SIP4-lacZ in different mutants and during growth on different carbon sources

Yeasts were grown in YNB/glucose (black bars) or YNB/galactose (grey bars) or derepressed in YP/ethanol (white bars) as described in the Experimental section. Data are means \pm S.D. from at least three independent experiments.

only $20 \%$ of that observed in a wild-type yeast. These results show that, while lack of Cat8 has a strong effect on transcription directed by either UAS1 or UAS2, Sip4 is dispensable for transcription directed by these UASs. They suggest also that different 


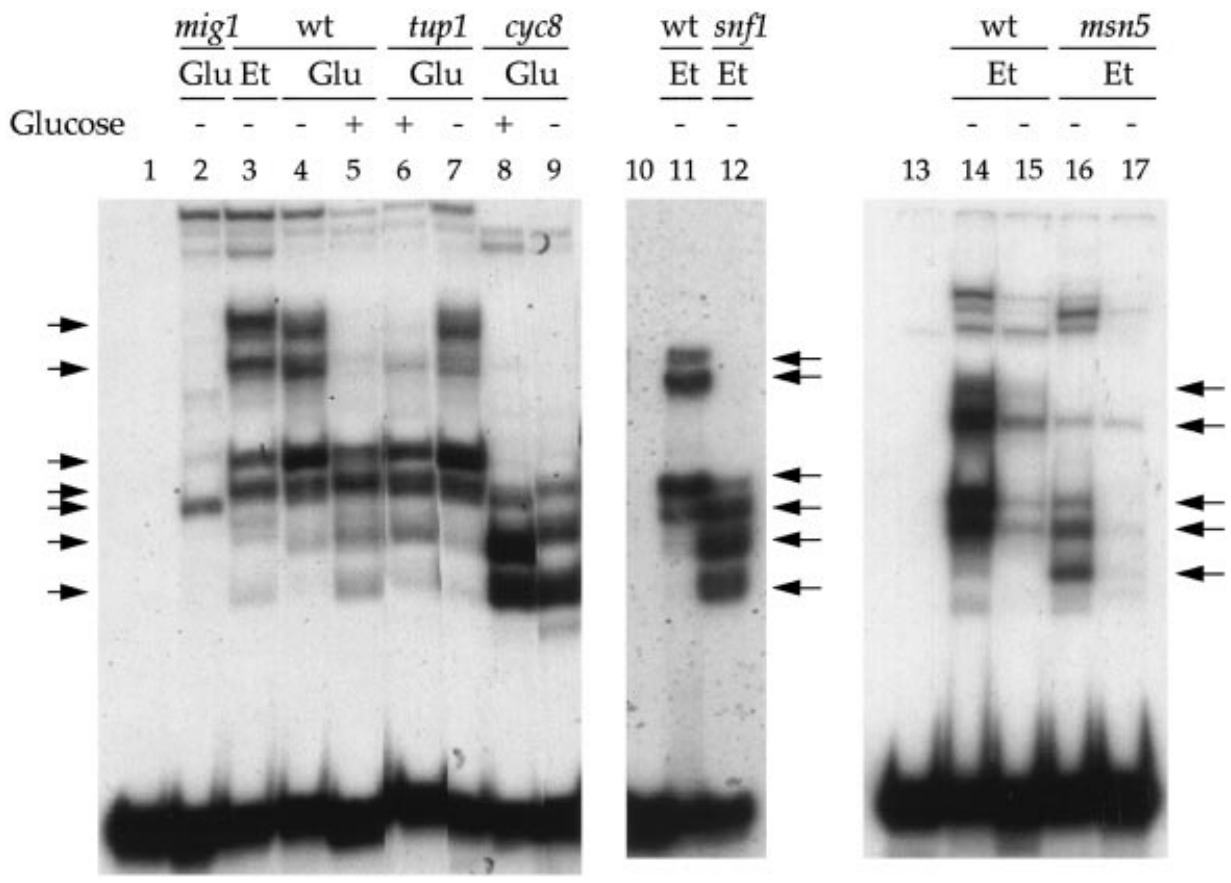

Figure 4 Band-shift assays with a URS sequence from the FBP1 promoter

Nuclear extracts were prepared from different yeast strains grown on YP/glucose (Glu) or derepressed in YP/ethanol (Et). Where indicated, $2 \%$ glucose was added to all the solutions used for the preparation of the nuclear extracts. In lanes 1-17 the URS1 oligonucleotide described in the Experimental section was used as a probe, and $20 \mu \mathrm{g}$ of the corresponding nuclear protein was added to samples in lanes 2-9, 11-12 and 14-17. In lanes 15 and 17 a 100-fold excess of unlabelled oligonucleotide was added to check the specificity of the interaction. Electrophoresis was performed using a polyacrylamide gel at $11 \%$. Specific complexes are indicated with arrows.

proteins may bind to the UASs in vivo and in vitro (see the Discussion).

\section{Expression of CAT8 and SIP4 is regulated in different ways}

Since both CAT8 and SIP4 are repressed during growth on glucose $[7,18,28]$, it may be expected that their expression is controlled by similar mechanisms. Repression of CAT8 by glucose has been reported to be markedly relieved in a mig1 mutant and only slightly so in a $h x k 2$ (hexokinase 2) mutant [7,28]. As shown in Figure 3, neither the hxk2 nor the migl mutations could relieve glucose repression of SIP4-lacZ; in a tup 1 mutant a weak increase in expression was seen in repressed cells. In our conditions, both the $h x k 2$ and the migl mutations relieved significantly the repression of CAT8-lacZ, the effect being stronger for $h x k 2$. Nevertheless, even in this case the expression reached in glucose was less than $5 \%$ of that achieved under derepressing conditions. On the other hand, in a tupl mutant glucose repressed $C A T 8$-lac $Z$ only about 3 -fold, as could be expected from the report that $C A T 8-l a c Z$ is only slightly sensitive to glucose repression in a miglmig 2 mutant [31]. We also tested the expression of the fusion genes $C A T 8-l a c Z$ and SIP4-lac Z during growth on galactose, a carbon source less repressing than glucose but able to block $F B P 1$ expression [32]. As shown in Figure 3, while the expression of SIP4-lacZ was only moderately increased under these conditions, $C A T 8-l a c Z$ expression was much higher than during growth on glucose, although it still remained significantly repressed.

\section{The capacity of URS1 to bind nuclear proteins and to repress transcription depends on the carbon source}

A sequence in the promoter of $F B P 1$, which corresponds to the consensus for binding the repressor Mig1 [12], has been shown in footprinting experiments to bind nuclear proteins [11]. To ascertain if the formation of DNA-protein complexes with this URS1 sequence was regulated by the carbon source, we conducted band-shift experiments with an oligonucleotide with the corresponding sequence and nuclear extracts from repressed and derepressed yeast cells. As shown in Figure 4 (lanes 3 and 4), the pattern of DNA-protein complexes was the same in both cases. However, since the preparation of nuclear extracts involves a $1 \mathrm{~h}$ incubation of the repressed yeast cells in a medium devoid of glucose, the previous result could be due to an experimental artifact. When we performed the band-shifts using nuclear extracts from repressed cells prepared by a procedure which included glucose in all the solutions used, the pattern of DNAprotein complexes was completely modified (Figure 4, lane 5). The two bands of low mobility disappeared and two bands of high mobility, barely visible with extracts from derepressed yeast, became stronger.

The multiplicity of bands observed could be related with the fact that Mig1 acts as a complex with the Cyc8 and Tup1 proteins $[13,33]$. We observed that the absence of Tup 1 in the band-shifts did not produce significant changes in the pattern of bands (Figure 4, lanes 6 and 7), but the lack of Cyc8 caused a very strong increase in the amount of the two complexes of higher mobility and complete disappearance of the complexes of lower mobility (Figure 4, lanes 8 and 9). In addition, in this case, the pattern of complexes formed was little affected by the presence or absence of glucose. These results suggest that the binding of Mig1 alone does not depend on the glucose in the medium, but that complexes including Cyc8 show a different behaviour when extracts of repressed or derepressed cells are used. It can be noted that a single specific DNA-protein complex of different mobility is still formed when extracts from a mig 1 mutant are used (Figure 4, lane 2). This complex is also ob- 


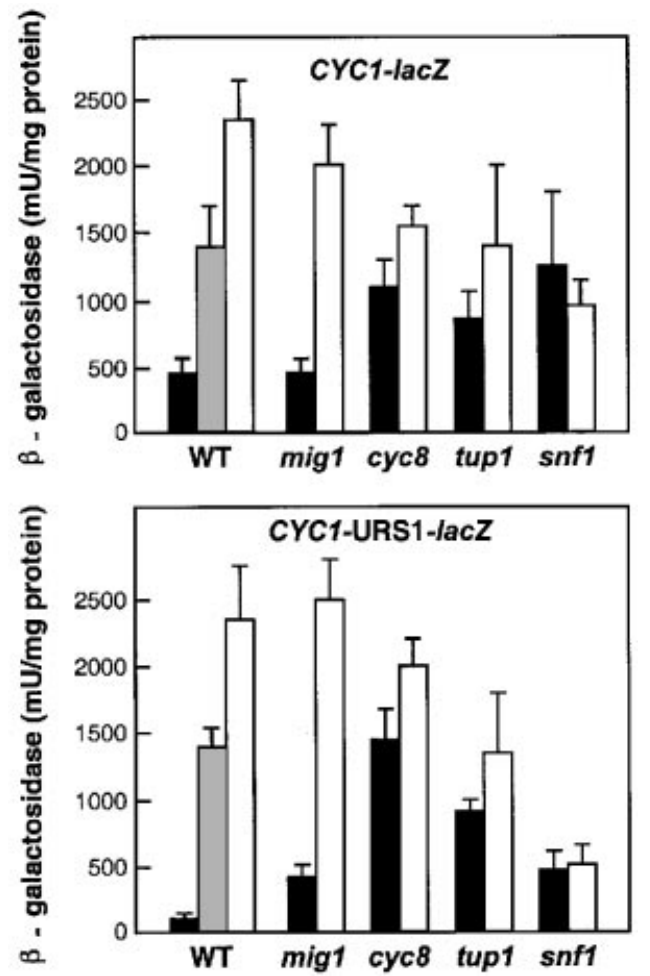

Figure 5 Expression of the fusion genes CYC1-lacZ and CYC1-URS1-lacZ in different media and in different mutants

The yeasts, transformed with pLG669-ZS or p0V31, were grown in YNB/glucose (black bars) or YNB/galactose (grey bars) or derepressed in YP/ethanol (white bars) as described in the Experimental section. Data are means \pm S.D. from at least three independent experiments.
A

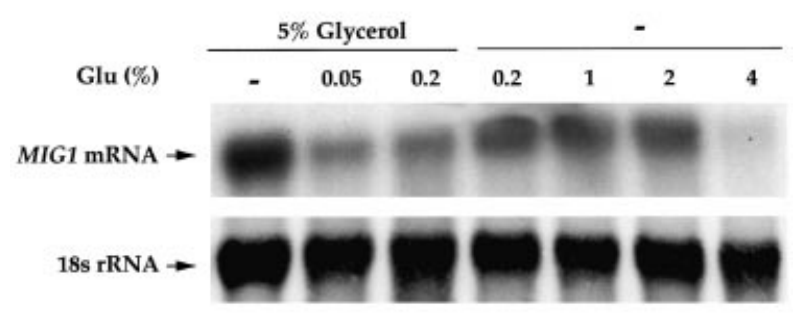

B

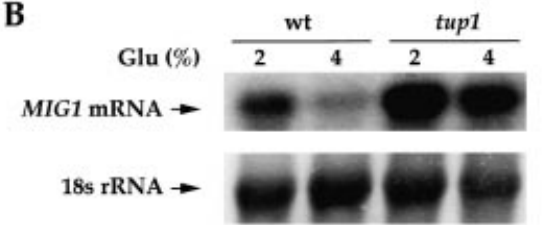

C

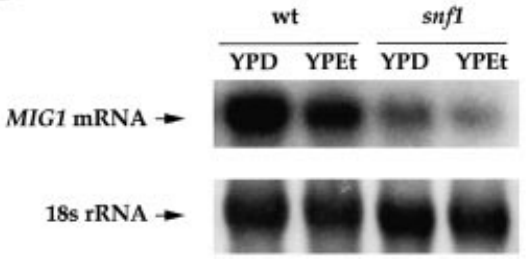

Figure 6 Influence of the carbon source on the levels of Mig1 mRNA

(A) Yeast strain S288C was grown in synthetic complete medium with the carbon sources indicated. (B) Strains W303-1A (wt) and L0Z021 (tup1) were grown in synthetic complete medium with 2 or $4 \%$ glucose, as indicated. (C) Strains W303-1A (wt) and H366 (snf1) were grown in YPD or derepressed in YP/ethanol. The yeasts were collected during the exponential phase of growth, and RNA was isolated and Northern analysis performed as described in the Experimental section.

To evaluate the in vivo behaviour of the URS1 sequence from the $F B P 1$ promoter, we inserted this sequence in a fusion gene CYC1-lacZ. The insertion caused a large decrease in the expression of this gene when the yeast was grown on glucose, but had no effect when the yeast was grown on galactose or derepressed in ethanol (Figure 5). Repression was completely relieved in a mig 1 mutant, a result which indicates that the protein other than Mig1 which may bind URS in vitro (Figure 4, lane 2) does not act as a repressor in vivo; repression during growth on glucose was also absent in $c y c 8$ or tup 1 mutants. On the other hand, in a snfl mutant the degree of repression was decreased to around 2-fold but became independent of the carbon source (Figure 5). Although the different mutations also affect the expression of CYC1-lacZ, it is clear that in the fusion gene URS1 had a negative effect in the presence of glucose and did not operate as an activator in any of the conditions tested.

\section{Mig1 mRNA levels are moderately regulated by the carbon source}

Since it was reported that the Mig1 mRNA level is not increased in cells grown on glucose [36], it has been assumed that MIG1 expression is constitutive. However, there is recent evidence for some control of $M I G 1$ expression by the carbon source: a $M I G 1$ lac $Z$ fusion was repressed over 10 -fold by $4 \%$ glucose, and this repression depended on Mig1 itself, together with the related repressor Mig2 [37]. As the only function described for Mig1 
Table 2 Influence of a low concentration of glucose on the derepression of fusion genes

Strain W303-1A was transformed with the reporter plasmids indicated (see the Experimental section) and grown in YNB medium with the carbon sources indicated. The yeasts were collected during the exponential phase of growth. Data are the means \pm S.D. from at least three determinations with different transformants. Gly, glycerol; Glu, glucose; Et, ethanol.

\begin{tabular}{lrccc}
\hline \multicolumn{5}{c}{$\beta$-Galactosidase activity (m-units/mg of protein) } \\
\cline { 2 - 5 } Fusion gene & $2 \%$ Glu & $0.2 \%$ Glu & $3 \%$ Gly $+2 \%$ Et $+0.2 \%$ Glu & $3 \%$ Gly $+2 \%$ Et \\
\hline FBP1-lacZ & $3 \pm 2$ & $5 \pm 1$ & $9 \pm 2$ & $1050 \pm 80$ \\
UAS1-lacZ & $23 \pm 1$ & $56 \pm 2$ & $220 \pm 8$ & $985 \pm 45$ \\
UAS2-lacZ & $19 \pm 2$ & $13 \pm 1$ & $29 \pm 1$ & $270 \pm 30$ \\
CAT8-lacZ & $2 \pm 1$ & $24 \pm 8$ & $74 \pm 23$ & $985 \pm 220$ \\
SIP4-lacZ & $15 \pm 6$ & $20 \pm 8$ & $29 \pm 5$ & $1090 \pm 250$ \\
& & & & \\
\hline
\end{tabular}

is performed precisely during growth on glucose, this observation appears surprising. We have reinvestigated the control of $M I G 1$ expression, using Northern analysis, comparing Mig1 mRNA levels during growth on different media (Figure 6A). While $4 \%$ glucose had a clear repressing effect (about 4-fold), mRNA levels were similar in the presence of $0.2 \%, 1 \%$ or $2 \%$ glucose. However, in a glycerol medium, the addition of as little as $0.05 \%$ glucose caused a decrease in the amount of Mig1 mRNA present. The repression produced by $4 \%$ glucose was relieved in a tup 1 mutant (Figure 6B), a result consistent with the observation that repression of $M I G 1$ depends on Mig1 and Mig2 [37].

Because no fluorescence could be detected in cells of a snf1 mutant carrying a GFP-MIG1 fusion (where GFP is green fluorescent protein), it has been suggested that $\mathrm{Snf} 1$ is required for $M I G 1$ expression [34]. We found, however, significant mRNA levels in a snfl strain grown in glucose (Figure 6C). It can be noted that when cells grown on YPD are derepressed in YP/ ethanol, there is a decrease in the Mig1 mRNA levels, in both the presence and absence of Snf1. This observation, together with the other results shown, indicates that control of the Mig1 mRNA levels is complex and that a straightforward cataboliterepression process cannot account for them.

\section{UAS1, UAS2 and URS1 respond differently to the concentration of glucose}

Among the large number of genes from $S$. cerevisiae regulated by glucose some are able to respond to low concentrations of glucose, while others require high concentrations to be turned on or off. For instance $H X T 2, H X T 3$ or $H X T 4$, which encode glucose transporters, and $S U C 2$, which encodes invertase, are induced by glucose in the range of $0.05-0.1 \%$, while the glucose transporter gene $H X T 1$ requires $2 \%$ glucose to be transcribed [38,39], and genes such as ICL1, ACS1 or MLS1, which encode proteins required under gluconeogenic conditions and which contain CSRE elements in their promoters, are fully derepressed at $0.2 \%$ glucose but are not transcribed at $2 \%$ glucose $[29,38,40,41]$. We did not detect fructose-1,6-bisphosphatase activity in yeast growing on $0.2 \%$ glucose, but this could be related to the capacity of glucose to trigger the proteolytic degradation of the enzyme [42]. However, under our experimental conditions (strains with W303 background growing on YNB with $0.2 \%$ glucose), there was also no expression of the fusion genes FBP1-lac $Z$ or UAS2-lac $Z$ and only a limited expression of UAS1-lac $Z$ (Table 2). To see if induction by a gluconeogenic carbon source is required in addition to the relief from glucose repression, we examined the expression of FBP1-lacZ, UAS1-
Table 3 Effect of the concentration of glucose in repression mediated by URS1 $_{F B P 1}$

Strain W303-1A was transformed with the reporter plasmids pLG669-ZS or p0V31 (see the Experimental section) and grown in YNB medium with glucose at the concentrations indicated. The yeasts were collected during the exponential phase of growth. Data are the means \pm S.D. from at least four experiments. Glu, glucose.

\begin{tabular}{lllll}
\hline \multicolumn{4}{c}{$\beta$-Galactosidase activity (m-units/mg of protein) } \\
\cline { 2 - 5 } Fusion gene & $0.2 \%$ Glu & $1 \%$ Glu & $2 \%$ Glu & $4 \%$ Glu \\
\hline CYC1-lacZ & $860 \pm 60$ & $460 \pm 85$ & $480 \pm 105$ & $450 \pm 20$ \\
CYC1-URS1-lacZ & $670 \pm 90$ & $210 \pm 30$ & $96 \pm 22$ & $100 \pm 20$ \\
\hline
\end{tabular}

lac $Z$ and UAS2-lac $Z$ in yeasts growing on $0.2 \%$ glucose, in the presence of ethanol and glycerol (Table 2). Under these conditions expression of FBP1-lacZ and UAS2-lac Z was still strongly repressed while UAS1-lac $Z$ was transcribed at $20-25 \%$ of the rate observed during growth on ethanol and glycerol (about 10fold induction). We have also examined the effect of a low concentration of glucose on the expression of CAT8-lac $Z$ and SIP4-lacZ (Table 2). There is a slight, but significant, derepression of CAT8-lacZ at $0.2 \%$ glucose, which increases in the presence of glycerol and ethanol, but remains at less than $10 \%$ of the values reached in the absence of glucose. In contrast, expression of SIP4-lacZ is only significantly increased in the complete absence of glucose.

Repression mediated by URS1 is also influenced by the concentration of glucose in the medium. As shown in Table 3, repression was strongest at $2 \%$ glucose, it decreased markedly at $1 \%$ and did not exceed $20 \%$ at $0.2 \%$ glucose. Thus the mechanisms which relay the glucose signal to UAS1, UAS2 and URS1 appear to have different sensitivities to glucose. Since expression of $M I G 1$ is partially repressed by $4 \%$ glucose, we wondered whether some protein alternative to Mig1 may be binding to URS1 in these conditions. This does not appear to be the case, since we found that URS1 did not cause any repression at $4 \%$ glucose in a migl background (results not shown).

\section{DISCUSSION}

We have studied different factors that affect the activity of the regulatory sites UAS1, UAS2 and URS1 of the FBP1 gene from $S$. cerevisiae. We have shown that the capacity of URS1 to repress transcription requires a high concentration of glucose in the medium (1-2\%), whereas expression directed by UAS1 or UAS2 is strongly decreased at $0.2 \%$ glucose. In Figure 7 we present a model for the regulation of FBP1. During growth on a gluconeogenic carbon source the protein kinase Snf1 is fully operative: it turns off the repressing protein Mig1 and turns on the transcriptional activators Cat8 and Sip4. Cat8 in turn is required for the transcription of SIP4 [10] and for activating the transcription of $F B P 1$ mediated by UAS1 and UAS2.

Since in a cat8 mutant expression of UAS1-lac $Z$ decreased strongly (Figure 2) while the formation of DNA-protein complexes between UAS1 and nuclear proteins was not affected (Figure 1), it appears that the synthesis of the protein(s) binding UAS1 in band-shift assays does not require Cat8, but that Cat8 is necessary for these proteins to be fully operative. Alternatively, in a cat 8 mutant ethanol metabolism could be compromised and this may affect the transcription and translation processes taking place after the shift of the culture to a YP/ethanol medium. The absence of DNA-protein complexes between UAS2 and nuclear 


\section{Ethanol, Glycerol}

\section{Low glucose, Galactose}

\section{High glucose}
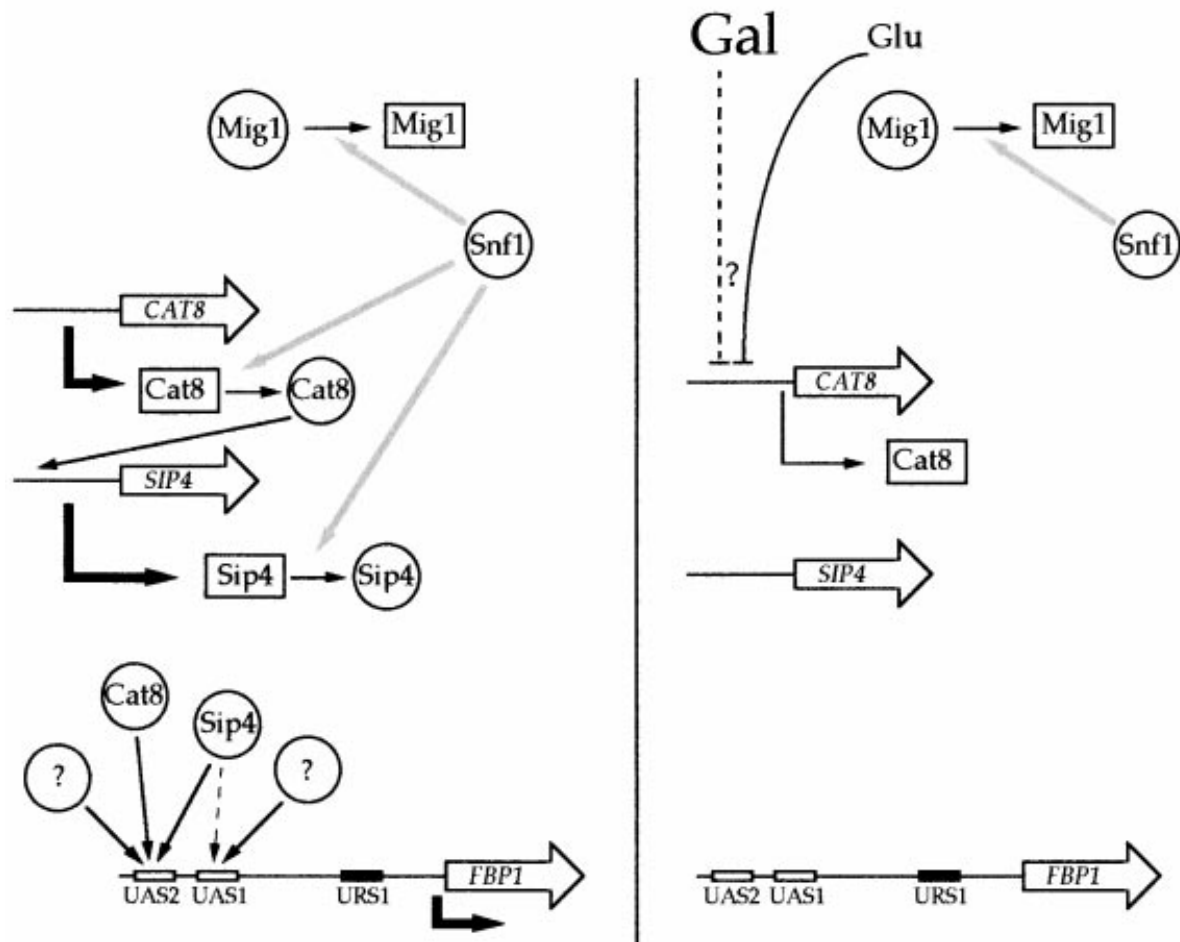

Transcriptional activation

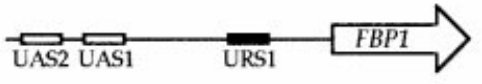

No expression
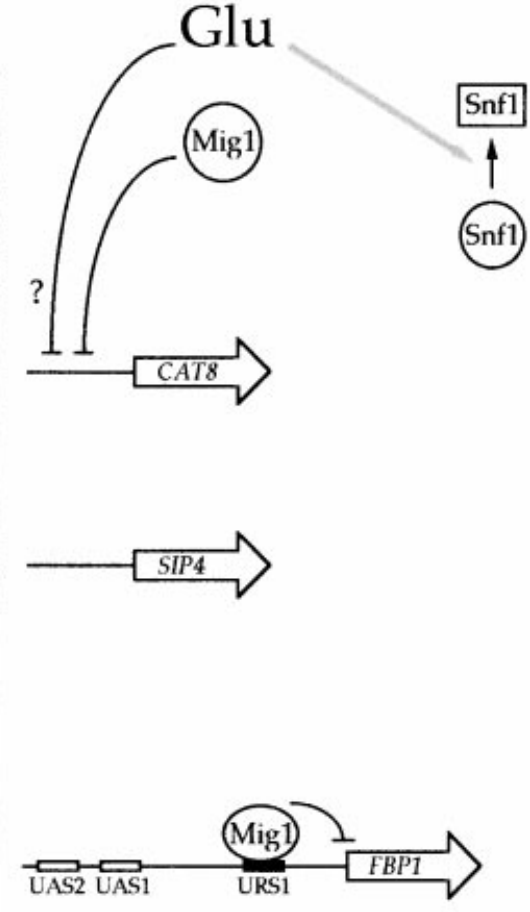

Transcriptional repression

Figure 7 Model for the regulation of FBP1 expression by the carbon source

Active proteins are shown as circles and inactive proteins as rectangles. Grey arrows indicate facilitation of a protein conformational change. For details see the text.

extracts from a sip4 mutant (Figure 1) appears contradictory with the high expression of the fusion gene UAS2-lacZ in this background (Figure 2). A possible interpretation is that different proteins (Cat8, Sip4 and perhaps another one, depending on both Cat8 and Sip4) are able to bind UAS2 in vivo, but that Cat8, at normal levels of expression, does not bind UAS 2 in vitro. Since Sip4 is not synthesized in the absence of Cat8 [10], neither cat8 nor sip4 extracts would allow the formation of DNA-protein complexes in vitro. On the other hand, the capacity of Sip4 (or an unidentified protein depending on Sip4) to activate transcription would be relatively low, and its absence, or down-regulation, in a sip4 mutant would allow the function of a different transcription factor (Cat8?) able to activate UAS2 more strongly.

In wild-type strains growing on galactose or low glucose (or in hxk2 mutants growing on high glucose), Snf1 is still able to inactivate Mig1 (Figure 7). However, in low glucose CAT8 expression is low (Table 2) and on galactose CAT8 is only partially derepressed (Figure 3). Under neither set of conditions is FBPI expressed, and there is only a slight derepression of SIP4 during growth on galactose; it is therefore likely that in the presence of sugars Cat 8 cannot be phosphorylated, a process required for its activity [43].

We observed that the operativity of URS1 in a fusion gene was dependent on the concentration of glucose (Table 3). The low level of repression, about $20 \%$, observed at $0.2 \%$ glucose is not due to lack of expression of $M I G 1$, since this gene is well expressed in media with low glucose (Figure 6). It is also unlikely that at low glucose Mig1 is mislocalized, since at $0.1 \%$ glucose most of the protein is in the nucleus [34]. It would seem therefore that an elevated glucose concentration is required for full activity of the Mig1 protein.

At high glucose Snf1 is inactive, Mig1 is in its active state and represses fully the expression of $C A T 8$ (Figure 7). The proteins that activate the transcription of SIP4 and $F B P 1$ are absent and the Mig1 complex binds URS1 and represses transcription. $F B P 1$ is therefore completely turned off. In some mutants such as hxk2, migl or tup 1, there is some expression of CAT8 in high glucose, but glucose appears to interfere with the phosphorylation of Cat8 [43] and there is therefore no expression of FBP1.

When we examined the effect of glucose on the formation of DNA-protein complexes with the URS1 element, we observed several strong complexes (Mig1-dependent) with nuclear extracts from both repressed and derepressed cells (Figure 4). Since Mig1 leaves the nucleus upon removal of glucose from the medium [34], only small amounts of Mig1 should be expected in nuclear extracts from derepressed cells. The abundance of URS1-binding proteins (and therefore presumably of Mig1) in these extracts is unlikely to be due to contamination by cytoplasmic material, as the extracts contained only small amounts of cytoplasmic proteins (results not shown). We hypothesize that when Mig1 exits from the nucleus it does not become free in the cytoplasm, but remains associated with some particulate fraction which co-sediments with the nucleus. Although expression of $M I G 1$ is controlled by the carbon source (Figure 6 and [37]), regulation of Mig1 occurs 
mainly through a phosphorylation catalysed by the protein kinase Snf1, in the absence of glucose [13,14,34,35,44,45]. Phosphorylation of Mig1 does not impair its binding to DNA, since strong DNA-protein complexes are formed with nuclear extracts from derepressed cells (Figure 4).

We interpret the multiplicity of URS1-protein complexes as follows. The pattern of two main bands found with extracts from a $c y c 8$ mutant may correspond to the binding of a single Mig1 molecule and to that of a Mig1 dimer. When Cyc8 is present it would bind to Mig1 and the bands will be shifted, although some complexes containing only Mig1 will still be formed. This pattern is seen with extracts from repressed cells or from a snfl mutant. With extracts from derepressed cells, there is a further large shift of the bands. The change in mobility is too marked to be accounted for simply by the phosphorylation of Mig1, but the phosphorylated Mig1 may be able to bind an additional protein, such as the Mig1 exportin, Msn5. The data with the $c y c 8$ mutant suggest that this binding depends also on the presence of Cyc8. On the other hand, none of the DNA-protein complexes formed in vitro appear to depend on Tup1.

The patterns we obtain in the band-shifts are different from those reported by $\mathrm{Wu}$ and Trumbly [46]. These differences may be related to the experimental conditions prevailing in the previous report: whole-cell extracts instead of nuclear extracts, glucose not included during the preparation of extracts from repressed cells, and the Mig1 binding site used, taken from $S U C 2$, different from the Mig1 binding site from the FBP1 gene.

We have shown that control through glucose of the different regulatory elements in the $F B P 1$ promoter takes place through different mechanisms. Different concentrations of glucose may activate different signalling pathways [47]. These pathways, however, have not been yet worked out. A factor that has been neglected up to now, but which is likely to be important in transmitting the signal of the presence of glucose, is the intracellular concentration of some intermediary metabolite(s). The picture that emerges from our results and those of others is that the control of transcription of $F B P 1$ is a complex process, involving the interaction of a multiplicity of elements. Although some of them have been identified, others remain to be understood.

We thank M. Carlson, K.-D. Entian, F. Estruch, L. Guarente, H. Ronne and R. J. Trumbly for providing plasmids or yeast strains, and C. Gancedo for critical reading of the manuscript. This work was supported by grants PB94-0091-C02-01 and PB971213-C02-01 from the Dirección General de Investigación Científica y Técnica. 0 . Z. had a fellowship from the Spanish Plan de Formación de Personal Investigador and 0 . V. was recipient of a fellowship from the DGICYT (Estancias temporales de científicos y tecnólogos extranjeros en España).

\section{REFERENCES}

1 Carlson, M. (1999) Glucose repression in yeast. Curr. Opin. Microbiol. 2, 202-207

2 Gancedo, J. M. (1998) Yeast carbon catabolite repression. Microbiol. Mol. Biol. Rev. 62, 334-361

3 Johnston, M. (1999) Feasting, fasting and fermenting. Glucose sensing in yeast and other cells. Trends Genet. 15, 29-33

4 Struhl, K. (1999) Fundamentally different logic of gene regulation in eukaryotes and prokaryotes. Cell 98, 1-4

5 Schöler, A. and Schüller, H.-J. (1994) A carbon source-responsive promoter element necessary for activation of the isocitrate lyase gene ICL1 is common to genes of the gluconeogenic pathway in the yeast Saccharomyces cerevisiae. Mol. Cell. Biol. 14, 3613-3622

6 Vincent, 0. and Gancedo, J. M. (1995) Analysis of positive elements sensititive to glucose in the promoter of the FBP1 gene from yeast. J. Biol. Chem. $\mathbf{2 7 0}$ 12832-12838

7 Hedges, D., Proft, M. and Entian, K.-D. (1995) CAT8, a new zinc cluster-encoding gene necessary for derepression of gluconeogenic enzymes in the yeast Saccharomyces cerevisiae. Mol. Cell. Biol. 15, 1915-1922
8 de Mesquita, J. F., Zaragoza, 0. and Gancedo, J. M. (1998) Functional analysis of upstream activating elements in the promoter of the FBP1 gene from Saccharomyces cerevisiae. Curr. Genet. 33, 406-411

9 Rahner, A., Hiesinger, M. and Schüller, H.-J. (1999) Deregulation of gluconeogenic structural genes by variants of the transcriptional activator Cat8p of the yeast Saccharomyces cerevisiae. Mol. Microbiol. 34, 146-156

10 Vincent, 0. and Carlson, M. (1998) Sip4, a Snf1 kinase-dependent transcriptional activator, binds to the carbon source-responsive element of gluconeogenic genes. EMBO J. 17, 7002-7008

11 Mercado, J. J., Vincent, 0. and Gancedo, J. M. (1991) Regions in the promoter of the yeast FBP1 gene implicated in catabolite repression may bind the product of the regulatory gene MIG1. FEBS Lett. 291, 97-100

12 Lundin, M., Nehlin, J. O. and Ronne, H. (1994) Importance of a flanking AT-rich region in target site recognition by the GC box-binding zinc finger protein MIG1. Mol. Cell. Biol. 14, 1979-1985

13 Treitel, M. A. and Carlson, M. (1995) Repression by SSN6-TUP1 is directed by MIG1, a repressor/activator protein. Proc. Natl. Acad. Sci. U.S.A. 92, 3132-3136

14 Östling, J. and Ronne, H. (1998) Negative control of the Mig1p repressor by Snf1pdependent phosphorylation in the absence of glucose. Eur. J. Biochem. 252, 162-168

15 Zaragoza, O., Lindley, C. and Gancedo, J. M. (1999) Cyclic AMP can decrease expression of genes subject to catabolite repression in Saccharomyces cerevisiae. J. Bacteriol. 181, 2640-2642

16 Lafuente, M. J., Gancedo, C., Jauniaux, J. C. and Gancedo, J. M. (2000) Mth1 receives the signal given by the glucose sensors Snf3 and Rgt2 in Saccharomyces cerevisiae. Mol. Microbiol. 35, 161-172

17 Rose, M. D., Winston, F. and Hieter, P. (1990) Methods in Yeast Genetics, Cold Spring Harbor Press, Cold Spring Harbor, NY

18 Lesage, P., Yang, X. and Carlson, M. (1996) Yeast SNF1 protein kinase interacts with SIP4, a $C_{6}$ zinc cluster transcriptional activator: a new role for SNF1 in the glucose response. Mol. Cell. Biol. 16, 1921-1928

19 Guarente, L. and Ptashne, M. (1981) Fusion of Escherichia coli lacZ to the cytochrome $c$ gene of Saccharomyces cerevisiae. Proc. Natl. Acad. Sci. U.S.A. 78 2199-2203

20 Trumbly, R. J. (1988) Cloning and characterization of the CYC8 gene mediating glucose repression in yeast. Gene $\mathbf{7 3}, 97-111$

21 Berben, G., Dumont, J., Gilliquet, V., Bolle, P. A. and Hilger, F. (1991) The YDp plasmids: a uniform set of vectors bearing versatile gene disruption cassettes for Saccharomyces cerevisiae. Yeast 7, 475-477

22 Williams, F. E. and Trumbly, R. J. (1990) Characterization of TUP1, a mediator of glucose repression in Saccharomyces cerevisiae. Mol. Cell. Biol. 10, 6500-6511

23 Chomczynscki, P. and Sacchi, N. (1987) Single-step method of RNA isolation by acid guanidium thiocyanate-phenol-chloroform extraction. Anal. Biochem. 162, 156-159

24 Feinberg, A. P. and Vogelstein, B. (1983) A technique for radiolabeling DNA fragments to high specific activity. Anal. Biochem. 132, 6-13

25 Miller, J. H. (1972) Experiments in Molecular Genetics, Cold Spring Harbor Press, Cold Spring Harbor, NY

26 Funayama, S., Gancedo, J. M. and Gancedo, C. (1980) Turnover of yeast fructosebisphosphatase in different metabolic conditions. Eur. J. Biochem. 109, 61-66

27 Schneider, R. I., Gander, I., Müller, V., Mertz, R. and Winnacker, E. L. (1986) A sensitive and rapid assay for nuclear factor I and other DNA-binding proteins in crude nuclear extracts. Nucleic Acids Res. 14, 1303-1317

28 Rahner, A., Schöler, A., Martens, E., Gollwitzer, B. and Schüller, H.-J. (1996) Dual influence of the yeast Cat1p (Snf1p) protein kinase on carbon source-dependent transcriptional activation of gluconeogenic genes by the regulatory gene CAT8. Nucleic Acids Res. 24, 2331-2337

29 Caspary, F., Hartig, A. and Schüller, H.-J. (1997) Constitutive and carbon sourceresponsive promoter elements are involved in the regulated expression of the Saccharomyces cerevisiae malate synthase gene MLS1. Mol. Gen. Genet. 255, 619-627

30 Proft, M., Kötter, P., Hedges, D., Bojunga, N. and Entian, K.-D. (1995) CAT5, a new gene necessary for derepression of gluconeogenic enzymes in Saccharomyces cerevisiae. EMBO J. 14, 6116-6126

31 Bojunga, N. and Entian, K.-D. (1999) Cat8p, the activator of gluconeogenic genes in Saccharomyces cerevisiae, regulates carbon source-dependent expression of NADPdependent cytosolic isocitrate dehydrogenase (Idp2p) and lactate permease (Jen1p). Mol. Gen. Genet. 262, 869-875

32 Rodríguez, C. and Gancedo, J. M. (1999) Glucose signaling in yeast is partially mimicked by galactose and does not require the Tps1 protein. Mol. Cell Biol. Res. Commun. 1, 52-58

33 Tzamarias, D. and Struhl, K. (1994) Functional dissection of the yeast Cyc8-Tup1 transcriptional co-repressor complex. Nature (London) 369, 758-761

34 DeVit, M. J., Waddle, J. A. and Johnston, M. (1997) Regulated nuclear translocation of the Mig1 glucose repressor. Mol. Biol. Cell 8, 1603-1618 
35 DeVit, M. J. and Johnston, M. (1999) The nuclear exportin Msn5 is required for nuclear export of the Mig1 glucose repressor of Saccharomyces cerevisiae. Curr. Biol. 9. 1231-1241

36 Nehlin, J. O. and Ronne, H. (1990) Yeast MIG1 repressor is related to the mammalian early growth response and Wilm's tumour finger proteins. EMBO J. 9 2891-2898

37 Lutfiyya, L. L., Iyer, V. R., DeRisi, J., DeVit, M. J., Brown, P. O. and Johnston, M. (1998) Characterization of three related glucose repressors and genes they regulate in Saccharomyces cerevisiae. Genetics 150, 1377-1391

38 Özcan, S. and Johnston, M. (1995) Three different regulatory mechanisms enable yeast hexose transporter (HXT) genes to be induced by different levels of glucose. Mol. Cell. Biol. 15, 1564-1572

39 Özcan, S., Vallier, L. G., Flick, J. S., Carlson, M. and Johnston, M. (1997) Expression of the SUC2 gene of Saccharomyces cerevisiae is induced by low levels of glucose. Yeast 13, 127-137

40 Schöler, A. and Schüller, H.-J. (1993) Structure and regulation of the isocitrate lyase gene ICL1 from the yeast Saccharomyces cerevisiae. Curr. Genet. 23, 375-381

41 Kratzer, S. and Schüller, H.-J. (1995) Carbon source-dependent regulation of the acetyl-coenzyme A synthetase-encoding gene ACS1 from Saccharomyces cerevisiae. Gene 161, 75-79

42 Gancedo, J. M. and Gancedo, C. (1997) Gluconeogenesis and catabolite inactivation. In Yeast Sugar Metabolism (Zimmermann, F. K. and Entian, K.-D., eds.),

pp. 359-377, Technomic Publishing Co., Lancaster

Received 29 January 2001/22 June 2001; accepted 26 July 2001
43 Rández-Gil, F., Bojunga, N., Proft, M. and Entian, K.-D. (1997) Glucose derepression of gluconeogenic enzymes in Saccharomyces cerevisiae correlates with phosphorylation of the gene activator Cat8p. Mol. Cell. Biol. 17, 2502-2510

44 Treitel, M. A., Kuchin, S. and Carlson, M. (1998) Snf1 protein kinase regulates phosphorylation of the Mig1 repressor in Saccharomyces cerevisiae. Mol. Cell. Biol. 18, 6273-6280

45 Smith, F. C., Davies, S. P., Wilson, W. A., Carling, D. and Hardie, D. G. (1999) The SNF1 kinase complex from Saccharomyces cerevisiae phosphorylates the transcriptional repressor protein Mig1p in vitro at four sites within or near regulatory domain 1. FEBS Lett. 453, 219-223

$46 \mathrm{Wu}, \mathrm{J}$. and Trumbly, R. J. (1998) Multiple regulatory proteins mediate repression and activation by interaction with the yeast Mig1 binding site. Yeast 14, 985-1000

47 Yin, Z., Hatton, L. and Brown, A. J. (2000) Differential post-transcriptional regulation of yeast mRNAs in response to high and low glucose concentrations. Mol. Microbiol. 35, 553-565

48 Thomas, B. J. and Rothstein, R. (1989) Elevated recombination rates in transcriptionally active DNA. Cell $\mathbf{5 6}, 619-630$

49 Mortimer, R. K. and Johnston, J. R. (1986) Genealogy of principal strains of the yeast genetic stock center. Genetics 113, 35-43

50 Alepuz, P., Matheos, D., Cunningham, K. and Estruch, F. (1999) The Saccharomyces cerevisiae RanGTP-binding protein Msn5p is involved in different signal transduction pathways. Genetics 153, 1219-1231 